\title{
Design and development of a 1-axis attitude control testbed for functional testing of EIRSAT-1
}

Rachel Dunwoody ${ }^{\mathrm{a} *}$, Joseph Thompson ${ }^{\mathrm{b}}$, Daire Sherwin ${ }^{\mathrm{b}}$, Maeve Doyle ${ }^{\mathrm{a}}$, Masoud Emam ${ }^{\mathrm{b}}$, Jessica Erkal ${ }^{\mathrm{a}}$, Joe Flanagan $^{\mathrm{b}}$, Gianluca Fontanesi ${ }^{\mathrm{b}}$, Andrew Gloster ${ }^{\mathrm{c}}$, Joseph Mangan ${ }^{\mathrm{a}}$, David Murphy ${ }^{\mathrm{a}}$, Favour Okosun ${ }^{\mathrm{b}}$, Conor O'Toole ${ }^{\mathrm{c}}$, Rakhi Rajagopalan Nair ${ }^{\mathrm{b}}$, Jack Reilly"a, Lána Salmonª, Sarah Walsha ${ }^{\mathrm{a}}$, Paul Cahill ${ }^{\mathrm{b}}$, Umair Javaid ${ }^{\mathrm{b}}$, Daithí de Faoite $^{\mathrm{b}}$, William O'Connor ${ }^{\mathrm{b}}$, Kenneth Stanton ${ }^{\mathrm{b}}$, Alexei Ulyanov ${ }^{\mathrm{a}}$, Ronan Wall ${ }^{\mathrm{a}}$, Lorraine Hanlon ${ }^{\mathrm{a}}$, Sheila McBreen ${ }^{\mathrm{a}}$, David McKeown ${ }^{\mathrm{b}}$

\author{
a School of Physics, University College Dublin, Belfield, Dublin 4, Ireland, rachel.dunwoody@ucdconnect.ie \\ ${ }^{\mathrm{b}}$ School of Mechanical and Materials Engineering, University College Dublin, Belfield, Dublin 4, Ireland \\ ' School of Mathematics and Statistics, University College Dublin, Belfield, Dublin 4, Ireland
}

* Corresponding Author

\begin{abstract}
The performance of the Attitude Determination and Control Subsystem (ADCS) of a CubeSat relies on consistent and robust inputs from sensors to provide the actuation to manoeuvre and stabilise the satellite orientation in space. This paper details the design and manufacture of a 1-axis motorised testbed to perform pre-flight ADCS functional testing of a nanosatellite based on the CubeSat Standard. This testbed has been developed to support the Educational Irish Research Satellite, EIRSAT-1, a 2U CubeSat being developed in University College Dublin (UCD) as part of the European Space Agency (ESA) Fly Your Satellite! (FYS!) Programme. EIRSAT-1 is a student led project to develop, build, test and launch Ireland's first satellite. The project is a collaborative effort of staff and students across a range of disciplines including physics, engineering and maths.
\end{abstract}

The design of the testbed allows all axes of the CubeSat to be tested individually. The design can be adapted easily to accommodate individual subsystem boards, such as an ADCS motherboard, in addition to larger CubeSat sizes, thus making it applicable to other missions. This testbed will be used to fully assess the functionality of the EIRSAT-1 ADCS motherboard, its inertial measurement unit, sun sensors, and magnetorquer actuation, first testing the Engineering Qualification Model (EQM) and then the Flight Model (FM). The testbed allows for polarity and performance checks of the sensors by comparison with known good reference sensor values. A controllable motorised rotating testbed allows for automated testing of the gyroscope and magnetometer. The performance of the five magnetorquers required for actuation is evaluated by an external magnetometer for each actuator. An easily adjustable artificial sun source allows for characterisation of the fine and coarse sun sensors response to change in angle relative to source. The testbed allows the satellite sensor and actuator outputs to be compared pre and post test procedures including ambient, vibrational and environmental test campaigns, in order to confirm full functionality or clearly indicate any issues.

Keywords—CubeSat, Attitude determination and control, Fly Your Satellite, EIRSAT-1

\section{INTRODUCTION}

The attitude determination and control subsystem of a satellite obtains the satellite's orientation in space and is responsible for spacecraft pointing. It is a challenge to verify this subsystem due to its numerous components and the complicated task of accurately recreating the space environment.

The attitude control subsystem of a satellite has three main components; the sensors, the actuators and the determination and control algorithm. Data from a suite of sensors is input into the attitude controller which runs an algorithm that fuses these sensor inputs together to determine the best estimate of the satellite's orientation. If the orientation of the satellite needs to be changed, the control system calculates the commands to send to the actuators to obtain the desired new orientation in space. Clyde Space Ltd. (CS) are providing the ADCS hardware for EIRSAT-1, as well as a bespoke ADCS algorithm which is currently under development and is expected to be delivered in September 2019. Therefore, the work presented is focused on validating only hardware components of the ADCS subsystem of EIRSAT-1.

This paper will outline the educational process of designing a versatile single axis testbed to verify that the individual components of the attitude control subsystem of EIRSAT- 1 are functional and to assess their performance. The design requirements included that the testbed can be modified to include other CubeSats from $1 \mathrm{U}$ to $3 \mathrm{U}$ and be relatively lowcost and robust, allowing for each axis of EIRSAT-1 to be tested in turn.

\section{EIRSAT-1}

\section{A. Mission Overview}

EIRSAT-1 is a $2 \mathrm{U}$ CubeSat $(10 \mathrm{~cm} \times 10 \mathrm{~cm} \times 22.7 \mathrm{~cm})$ being built in UCD as part of the ESA FYS! Programme. It is a student led project with students taking leadership roles including management of subsystems and payloads. 
The EIRSAT-1 project is driven by educational objectives to enable Irish students to gain sought-after skills for the space sector, foster collaboration with industry and inspire future generations in space science and engineering [1].

EIRSAT-1 consists of conventional CubeSat subsystems such as an On-Board Computer, Communications module, ADCS and an Electrical Power Subsystem (EPS) [2]. The EPS consists of four solar panels on the $+\mathrm{X},+\mathrm{Y},-\mathrm{X}$ and $-\mathrm{Y}$ faces of the satellite [2], the EPS motherboard and battery. All of these are being procured as 'commercial-off-the-shelf' (COTS) components from Clyde Space Ltd. In addition to these subsystems, three novel experiments will be flown on EIRSAT1, the Gamma-ray MODule (GMOD), the ENBIO MODule (EMOD) and Wave Based Control (WBC) [2].

\section{B. Experimental Payloads}

Gamma-ray Module: GMOD is a bespoke gamma-ray detector being designed and built in UCD. During the mission lifetime of EIRSAT-1 it will study Gamma-ray Bursts, high energy explosive events due to a neutron star merger event or the death of a massive star. GMOD is based on technologies that have been developed in UCD as part of previous work funded by ESA [3]. GMOD is expected to detect approximately 20 GRBs a year above a $10 \sigma$ threshold [2].

ENBIO Module: EIRSAT-1 is working in collaboration with the Irish company ENBIO Ltd. on the ENBIO Module. On the $+Z$ face of EIRSAT-1, there will be four thermal coupons, two will have an ENBIO Ltd. SolarWhite spacecraft surface treatment and the other two will be treated with SolarBlack. EIRSAT-1 will conduct in-flight performance monitoring of these thermal barrier coatings [2].

Wave Based Control: Wave Based Control is the third experiment on-board EIRSAT-1. It is a novel attitude control algorithm for flexible subsystems that has been developed in UCD. WBC has been tested extensively in a simulation environment but EIRSAT-1 will provide the first in space test of this algorithm [4].

\section{ATTITUDE DETERMINATION AND CONTROL SUBSYSTEM OF EIRSAT-1}

\section{A. Overview}

The ADCS of EIRSAT-1, hereafter ADCS, will be responsible for detumbling the satellite post deployment from the International Space Station and achieving stable pointing. The pointing direction of EIRSAT-1 is vital for charging the battery via the four solar panels while ensuring there is enough sunlight incident on the four EMOD thermal coupons. EIRSAT-1 will be spin stabilised about the z-axis with an angle of $58^{\circ}$ away from the sun and $90^{\circ}$ from Celestial North. The ADCS Motherboard (MB) provided by Clyde Space Ltd. will have a specific mode and state which will host the algorithm that will ensure these pointing requirements are fulfilled throughout the mission. The ADCS consists of the following components: ADCS
Motherboard, two 3-axis magnetometers (MTM), one 3-axis gyroscope, five coarse sun sensors (CSS), one fine sun sensor (FSS), GPS and the five magnetorquer (MTQ) actuators. The location and function of each of the ADCS components are presented in Table 1.

The 1-D testbed design is required to take into consideration these different components of the attitude control subsystem and determine a method of testing the basic functionality of the suite of sensors and set of magnetic actuators.

\begin{tabular}{|c|c|c|c|}
\hline $\begin{array}{c}\text { ADCS } \\
\text { Component } \\
\end{array}$ & Quantity & Location & Function \\
\hline $\bar{A} A D C S M B$ & $\overline{11}$ & $\begin{array}{l}\text { PCB Stack of } \\
\text { EIRSAT-1 }\end{array}$ & $\begin{array}{l}\text { Record sensor values } \\
\text { and use in attitude } \\
\text { control algorithm to } \\
\text { point satellite via } \\
\text { magnetic actuators }\end{array}$ \\
\hline$M T Q$ & 5 & $\begin{array}{l}+\mathrm{X},+\mathrm{Y},-\mathrm{X},-\mathrm{Y} \\
\text { Solar panels, } \\
\mathrm{Z} \text {-axis MTQ in } \\
\text { PCB stack }\end{array}$ & $\begin{array}{l}\text { Actuators to change } \\
\text { attitude of EIRSAT- } 1\end{array}$ \\
\hline FSS & 1 & $\begin{array}{l}+Z \text { face of } \\
\text { EIRSAT- } 1\end{array}$ & $\begin{array}{l}\text { Provide two sun } \\
\text { angles for attitude } \\
\text { control algorithm }\end{array}$ \\
\hline CSS & 5 & $\begin{array}{c}+\mathrm{X},+\mathrm{Y},-\mathrm{X},-\mathrm{Y} \\
\text { Solar panels, } \\
-\mathrm{Z} \text { face }\end{array}$ & $\begin{array}{l}\text { Coarse position of } \\
\text { sun via increase in } \\
\text { analog output when } \\
\text { illuminated }\end{array}$ \\
\hline GPS & 1 & $\begin{array}{l}+\mathrm{X} \text { face of } \\
\text { satellite }\end{array}$ & $\begin{array}{l}\text { Time and satellite } \\
\text { position }\end{array}$ \\
\hline $\begin{array}{l}\text { 3-axis } \\
\text { MTM }\end{array}$ & 2 & ADCS MB & $\begin{array}{l}\text { Provide readings of } \\
\text { local magnetic field } \\
\text { to ADCS MB }\end{array}$ \\
\hline $\begin{array}{c}\text { 3-axis } \\
\text { Gyroscope }\end{array}$ & 1 & ADCS MB & $\begin{array}{c}\text { Provide angular } \\
\text { velocity to ADCS } \\
\text { MB }\end{array}$ \\
\hline
\end{tabular}

Table 1: Components of EIRSAT-1 ADCS

The electrical specifications of the ADCS MB require a $5 \mathrm{~V}$ and $3.3 \mathrm{~V}$ power supply to be provided. The $5 \mathrm{~V}$ bus is expected to draw $90 \mathrm{~mA}$ if the MTQs are not driven and the $3.3 \mathrm{~V}$ bus is expected to draw $\leq 1 \mathrm{~mA}$ [5]. These requirements and the dimensional specifications of the ADCS MB were important to consider for the mounting of a single PCB to the single-axis testbed.

\section{B. I2C Communication Protocol}

As part of the software development for the testbed, it was essential to account for the communication protocol of the ADCS MB. The ADCS MB communicates via I2C. The OBC or operating computer is the master and the ADCS MB is the slave with address $0 \times 41$ [5]. In addition to providing power, two wires are required for communication, the I2C Data line and the I2C Clock. The ADCS MB provided by CS contains two features to make the $\mathrm{I} 2 \mathrm{C}$ communication more robust and mitigate the effects of radiation damage due to single event upsets such as cosmic ray interacting with the ADCS MB. 
Each time a register on the ADCS MB is written to, an 8-bit address hash (AHS) must be provided. If the AHS does not match that of the field being written to, then no data will be written to that memory location. Between the AHS and data being written to the field there is an 8-bit Forward Error Correction (FEC) field. Bits 7 and 6 are used to indicate if the FEC field is in use, and bits 5 to 0 are obtained using an error correcting linear code which must be calculated using the specific data that is being sent [5]. This code can be used to ensure that the data being received from the ADCS MB has not been corrupted.

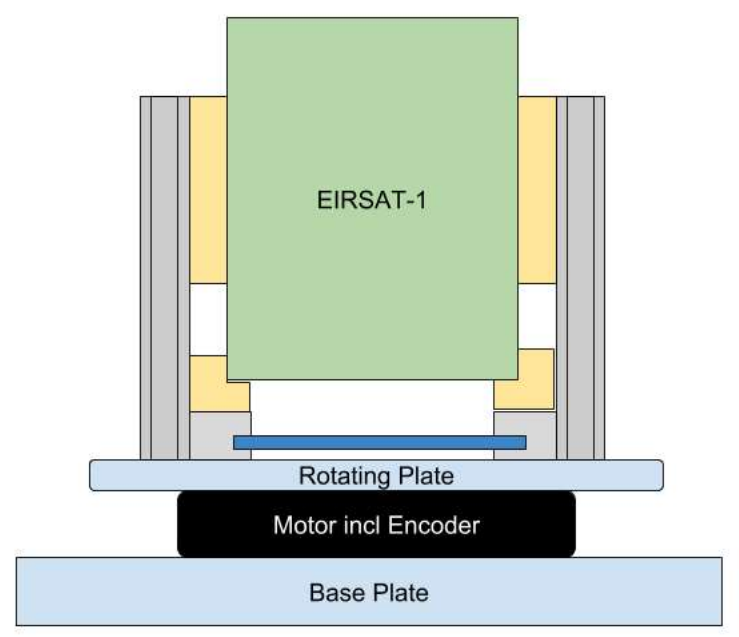

Fig. 1. Initial design concept for single axis testbed based on aluminium extrusions and 3D printed supports

The CS Interface Control Document for the ADCS MB also contains a set of conversion equations [5]. These equations need to be implemented in the software to transform the raw outputs from the ADCS MB sensors and actuators into physical units.

\section{Single AXIs Testbed Design}

\section{A. Design Requirements}

The single axis testbed requirements are governed by the ADCS components of EIRSAT-1 while also allowing versatility in the design for future use. In order to assess the ADCS MB, EQM and FM [6], the testbed had to fulfil the following:

- Allow motorised rotation about 1-axis with control of angular velocity and position

- Provide a method of monitoring the rotational rate of the testbed for comparison with the on-board gyroscope

- Support EIRSAT-1 in horizontal and vertical orientation and support single PCBs of a CubeSat Standard
- Incorporate reference magnetometers for comparison with the two MTMs on the ADCS MB and to assess the five magnetorquer actuators

- Possess a fake sun source mounted to illuminate CSS and FSS from different orientations to test the sun sensors

\section{B. Design Outline}

In Fig 1, the main hardware components of the single axis testbed are presented. A motor is secured to a baseplate and then a second plate is attached on top of the motor to hold the equipment being tested. To ensure compatibility with different orientations of EIRSAT-1 and even future missions of $1 \mathrm{U}$ to $3 \mathrm{U}$ standards, a versatile mounting structure on the rotating plate was designed.

Aluminum extrusion is mounted directly to the rotating plate to provide the main structure. The yellow components in Fig 1 present the initial concept of the 3D printed plastic extrusions to be in contact with any flight hardware and allow for versatility in the mounting of hardware components to the testbed. Currently plastic extrusions for the vertical support of EIRSAT-1, as in Fig 2, the horizontal support and for solely the ADCS MB have been designed and successfully printed. These components were printed in nylon as it is low cost and has a lower coefficient of friction than the conventional 3D printer material, Polylactic Acid (PLA).

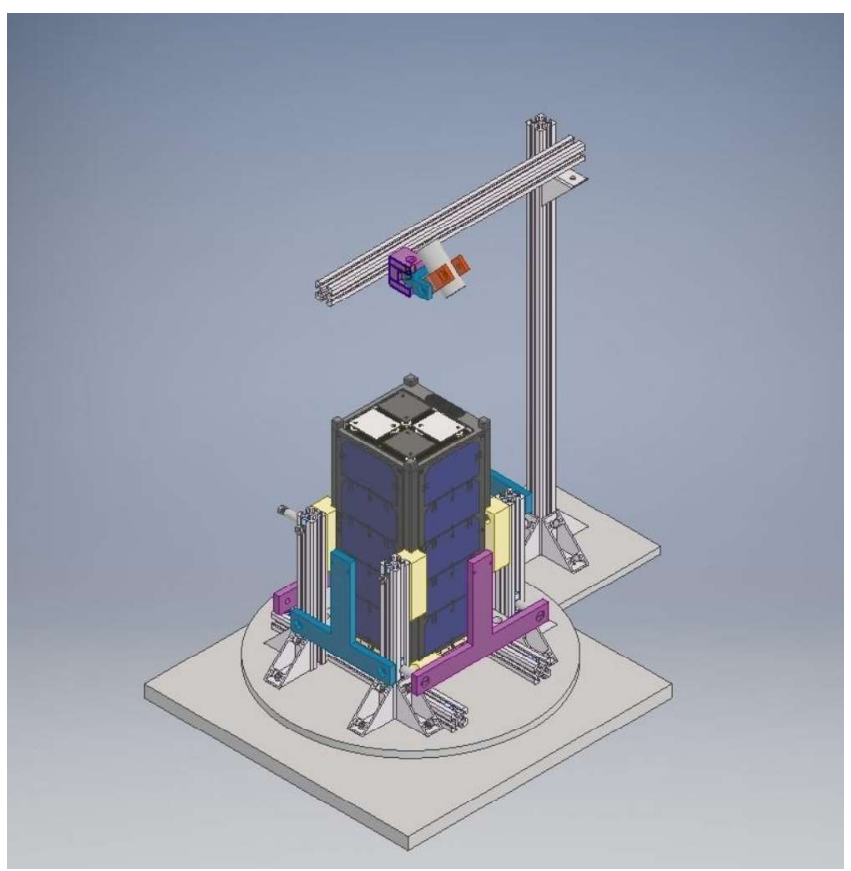

Fig. 2. Final CAD model for single axis testbed including rotating testbed and sun source support structure

Additional components were added to the testbed design to test the different sensors and actuators that comprise the EIRSAT1 attitude control subsystem. Fig 2 presents the final Computer Aided Design (CAD) model for the single axis testbed 
including the final design for the support stand for the sun source mimic. To allow for testing of the magnetorquers on each face of the satellite and the magnetometers on the ADCS $\mathrm{MB}$, five reference magnetometers are mounted on the testbed. An encoder was selected to measure the angular velocity of the motor for comparison with the ADCS MB gyroscope. In addition to the single axis testbed, a separate sun source mimic support stand was designed to facilitate the testing of the five coarse sun sensors on board EIRSAT-1 and the fine sun sensor on the $+Z$ face.

The iFlight iPower Motor GBM110-150T was selected for the single axis testbed to provide a low maintenance and high durability motor. This motor put constraints on the design of the testbed. The base plate and rotating plate are mounted directly to the motor as it has four mounting screw holes on its base and eight on its upper face. The motor also accommodates a 12-wire slip ring allowing for $5 \mathrm{~V}, 3.3 \mathrm{~V}$ and GND to be connected to the ADCS MB or satellite, as well as the clock and data lines for the $\mathrm{I} 2 \mathrm{C}$ bus communication. The motor controller procured for the testbed was the Odrive V3.5 [7] as it is a versatile controller that is suitable for brushless DC motor control and has open source hardware and software. The Odrive motor controller supports many interfaces including USB to computer or Raspberry Pi and UART to Arduino, both of which were used during the development of the testbed. The Odrive has support for a set of encoders that can be used alongside the motor. This governed the selection of the encoder for the testbed.

The encoder selected to measure the angular velocity of the motor was the AS5047P on-axis magnetic rotary encoder with a resolution of 4000 steps or 1000 pulses per revolution meaning an angular resolution of $0.09^{\circ}$ [8].

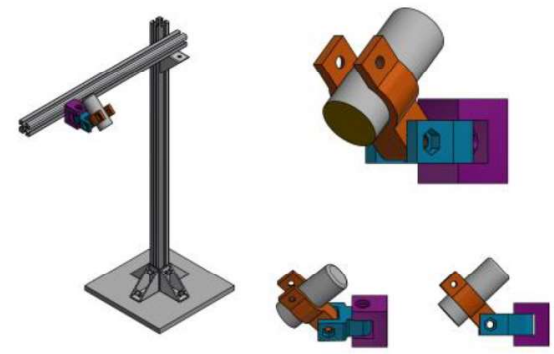

Fig.3 Left: Sun source mimic support stand. Right: Three 3D printed components for wide range of incident angles of sun mimic on sun sensors

The other electronic hardware of the testbed was based on the Grove System which dictated the choice of reference magnetometer [9]. The Grove system is a connector prototyping scheme for simple connection and modification of sensors to a base microprocessor. An Arduino UNO obtains readings from five Grove 3-axis Digital Compass Units which each host a BOSCH BMM150 Three-axis Geomagnetic Sensor. An Adafruit $\mathrm{I} 2 \mathrm{C}$ multiplexer is required as all the devices have the same I2C address [10]. A specific byte commands the I2C Multiplexer to communicate to the corresponding sensor, so only one MTM can be communicated with at a time, but as the ADCS MB only updates the sensor values at $1 \mathrm{~Hz}$, this does not impact the comparison of the reference MTMs to the ADCS MB sensors.

The final set of sensors that need to be tested as part of the EIRSAT-1 ADCS are the sun sensors. In order to determine the functionality and performance of the five coarse sun sensors and one fine sun sensor on-board EIRSAT-1, a sun source mimic is required. Clyde Space Ltd. uses the Fenix LD20 in their test procedures for sun sensors [11]. As the LD20 was decomissioned, the upgraded model LD22 was purchased as it has similar properities to the LD20. To ensure the light output from the torch is constent during testing the battery powered device was converted to AC power including a switch.

A stand from aluminium extrusion was designed to support a $3 \mathrm{D}$ printed device to allow for a wide range of angles of incidence of the torch beam on the faces of the sun sensors on board the satellite. As in Fig 3, a combination of three components were used to allow for movement in two axes and nylon screws were used to secure the clamp components.

\section{RESULTS}

\section{A. Preliminary Design Assessment}

After the full assembly of the testbed, as in Fig. 4, some preliminary assessment was conducted to determine its performance capabilities.

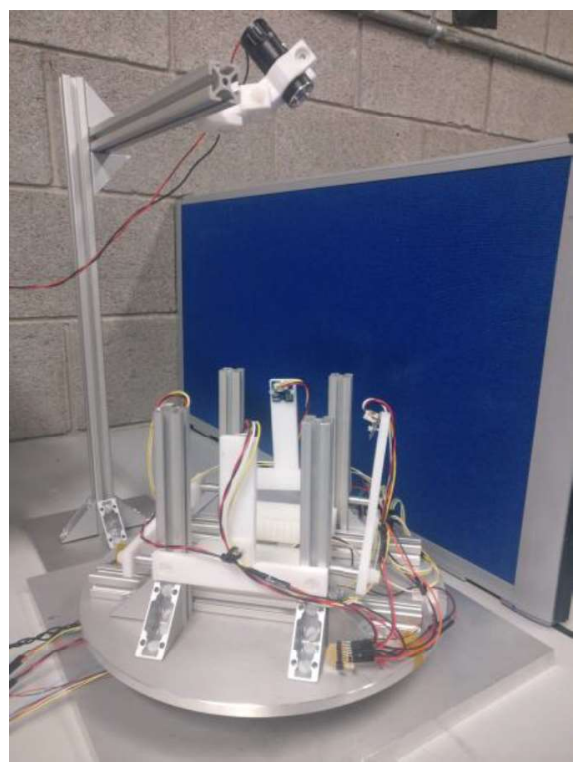

Fig.4. Assembled single axis testbed with sun support stand

The testbed design successfully fulfilled the initial requirements. The testbed allows for motorised rotation of a CubeSat or CubeSat Standard PCB about an individual axis. The concept of having 3D printed nylon components in contact with the flight hardware has resulted in a highly versatile design 
which can accommodate single boards and can be modified for $1 \mathrm{U}$ to $3 \mathrm{U}$ satellites. The encoder mounted under the motor allows for the rotational velocity of the motor to be measured for comparison with the ADCS MB gyroscope. The five reference magnetometers allow for analysis of the two magnetometers on the ADCS MB and the five magnetorquer actuators. Furthermore, the sun source support stand constructed as in Fig 3 facilitates the assessment of the coarse and fine sun sensors on board EIRSAT-1.

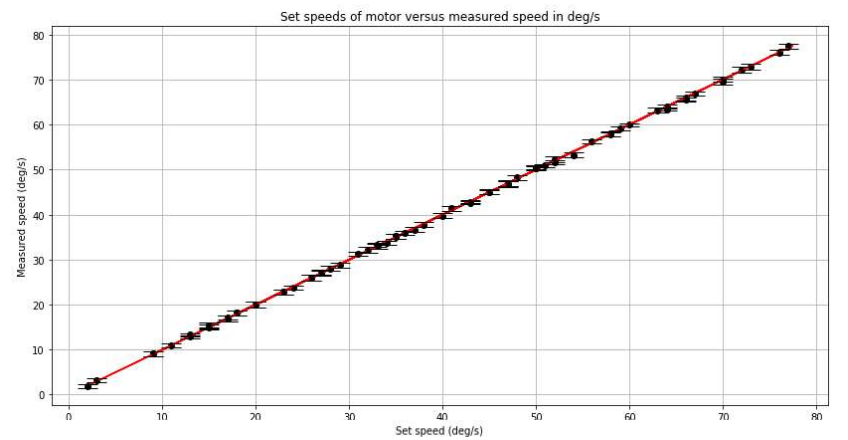

Fig.5. Set speed versus measured speed of single axis motorised testbed

Fig 5 shows the velocity control performance assessment of the testbed. The angular velocity set using the Odrive motor controller is compared with the actual measured angular velocity of the motor using the magnetic rotary encoder output. Each measured reading is the average of sixty readings taken over a period of sixty seconds. The assessment of the velocity control of the motor found a correlation between the two data sets using linear regression as $1.002 \pm 0.003$. A similar graph was constructed for the position control accuracy of the testbed which was found to be $1.018 \pm 0.016$. Both methods can be used to accurately control the velocity and position of the motorised testbed.

\section{B. ADCS MB Testing}

To date, basic acceptance testing has begun on the CS ADCS $\mathrm{MB}$. This involves ensuring the ADCS MB can communicate with each sensor and actuator provided by CS. The output of each sensor using the ADCS MB is also being used to verify the functionality of each component of the ADCS. Tests have been performed to ensure that the mode and state of the ADCS MB can be changed and that the time and positional data of the ADCS MB can be modified. These two features are important as the WBC payload needs to be implemented in test mode as it allows for commanding of the magnetorquers, and for the positional and time data to be accessed and altered. Once these test procedures are complete, the ADCS MB will be mounted to the single axis testbed described and the performance of the sensors on the motherboard will be assessed. After EQM integration, the ADCS components in the full system will be evaluated using the single axis testbed.

\section{CONCLUSION}

This paper has presented the design, construction and assessment of a single axis motorised testbed that can be used to analyze the performance of the sensors and actuators of the attitude control subsystem of $1 \mathrm{U}$ to $3 \mathrm{U}$ CubeSats. In the specific application to EIRSAT-1, the testbed has the capabilities of verifying the performance of the on-board gyroscope, magnetometers, sun sensors and magnetorquers. This testbed will be used for subsystem testing of the EQM and FM ADCS Motherboard of EIRSAT-1 and the EQM and FM of EIRSAT1 [6]. This project will provide a basis for future designs for testing the attitude control subsystem of EIRSAT-1, and other missions such as the addition of other sensor or actuator capabilities, and the development of the 3 -axis test rig.

\section{ACKNOWLEDGMENT}

The EIRSAT-1 team acknowledges support from ESA via PRODEX under contract number 4000124425. RD acknowledges the ESA's student sponsorship programme for funding the travel costs associated with the attendance of the symposium. SW acknowledges support from the ESA under PRODEX contract number 4000120713. DM acknowledges support from the Irish Research Council (IRC) under grant GOIPG/2014/453. JT acknowledges support from the IRC under grant GOIPG/2014/684. MD acknowledges support from the IRC under grant GOIP/2018/2564. JE, JR and RD acknowledge scholarships from the UCD School of Physics. AG acknowledge scholarships from the UCD School of Mathematics and Statistics. CO'T acknowledges support from the IRC under grant GOIPG/2017/1031. LS acknowledges support from the IRC under grant GOIPG/2017/1525. SMB, JM and AU acknowledge support from Science Foundation Ireland under grant number $17 / \mathrm{CDA} / 4723$.

\section{REFERENCES}

[1] J. Thompson et al., Double-dipole antenna deployment system for EIRSAT-1, 2U CubeSat. 2nd Symposium on Space Educational Activites. April 11-13 2018, Budapest, Hungary, 2018. Paper ID: SSEA-2018-78.

[2] D. Murphy et al., EIRSAT-1: The Educational Irish Research Satellite, 2nd Symposium on Space Educational Activities. April 11-13 2018, Budapest, Hungary. Paper ID: SSEA-2018-93

[3] A. Ulyanov et al., 2016. Performance of a monolithic LaBr3:Ce crystal coupled to an array of silicon photomultipliers. Nuclear Instruments and Methods in Physics Research Section A Accelerators Spectrometers Detectors and Associated Equipment. 810. 107-119.

[4] D. Sherwin et al Wave-based attitude control of EIRSAT-1, 2U CubeSat. 2nd Symposium on Space Educational Activites. April 11-13 2018, Budapest, Hungary, 2018. Paper ID: SSEA-2018-93.

[5] Clyde Space Ltd. ADCS Motherboard Interface Control Document: ICD25-01232 RevF, 2017a

[6] S. Walsh et al., Assembly, Integration and Verification Activities for a $2 \mathrm{U}$ CubeSat, EIRSAT-1, $20193^{\text {rd }}$ Symposium on Space Educational Activites, in press

[7] Oskar Weigl. Odrive Robotics, 2019. https://odriverobotics.com/ [Accessed: 13-05-2019]

[8] AS5047P Datasheet: 14-Bit On-Axis Magnetic Rotary Position Sensor with 12-Bit Decimal and Binary Incremental Pulse Count for 28krpm High Speed Capability [v1 01], ams AG, 2016

[9] Seed Technology Co. Ltd., Grove - 3-Axis Digitial Compass v2.0, 2018. http://wiki.seeedstudio.com/Grove-3-Axis_Digitial_Compass_v2.0/ [Accessed: 10-05-2019]

[10] Adafruit. Adafruit TCA9548A 1-to-8 I2C Multiplexer Breakout: Overview, 2015.https://learn.adafruit.com/adafruit-tca9548a-1-to-8-i2cmultiplexer-breakout/overview[Accessed: 10-05-2019]

[11] Clyde Space Ltd. ADCS Integration Test Procedure: TST-2149 Rev A, $2017 b$ 